\title{
Transient editing catches the eye
}

\author{
The preclinical performance of subretinal or intracorneal delivery of Cas9 nucleases encoded in RNA foreshadows \\ safer and effective one-and-done gene therapies for eye diseases.
}

C RISPR gene editing has many hurdles to clear before it can produce safe and effective therapies for human use. There are three outstanding challenges: performing edits in specific cells in particular tissues, minimizing off-target edits with potentially pathogenic consequences, and reducing the immunogenicity of the CRISPR nucleases. The delivery problem can be addressed more easily when the therapy can be administered directly to accessible anatomical regions. And the editing specificity can be improved with ever more faithful and reliable nucleases and with better designed RNA guides. Yet these two challenges can also benefit from opening a transient window of action - by encoding the editing machinery in the form of mRNA rather than DNA (mRNA is shorter-lived than plasmid DNA) - and by delivering it via viral vectors with beneficial tropism.

This strategy is exemplified in two articles by Yujia Cai and colleagues. In their Article in this issue of Nature Biomedical Engineering they describe an approach to knock out vascular endothelial growth factor A in retinal pigment epithelium. And using a similar approach they knocked out the UL8 and UL29 genes of the herpes simplex virus type 1 in corneal stroma (D. Yin et al. Nat. Biotechnol. https://doi. org/10.1038/s41587-020-00781-8; 2021). Disrupting the production of the growth factor slowed down the development of choroidal neovascularization in a mouse model of wet age-related macular degeneration, and deleting UL8 and UL29 blocked the replication of the virus in mouse models of herpetic stromal keratitis. In both studies, the transient activity of the DNA-cutting SpCas9 (Cas9 from Streptococcus pyogenes) aided by RNA guides for these genes markedly reduced choroidal neovascularization and viral recurrence (by reducing retrograde transport from viral reservoirs in the cornea to trigeminal ganglia). Hence, at least for these retinal and corneal conditions, effective therapeutic outcomes (and, potentially, cures) may not always require prolonged Cas 9 activity. For

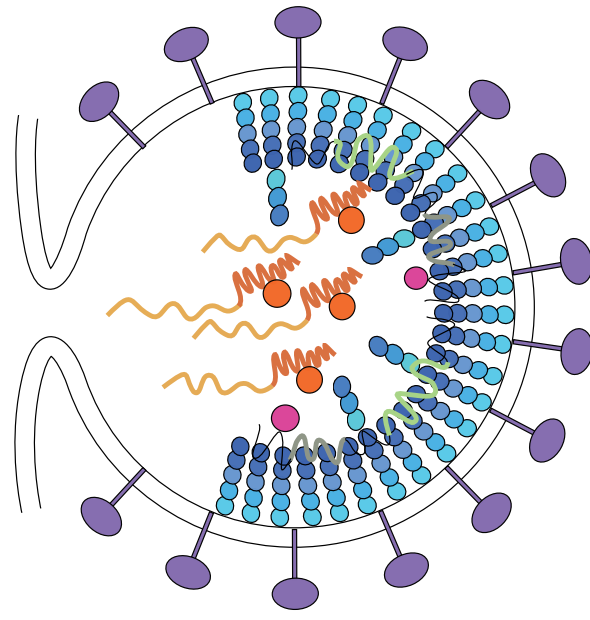

Figure adapted from the News \& Views article by F. Bubeck and D. Grimm.

many conditions, meaningful therapeutic outcomes may however only be achieved via sustained expression of the CRISPR nuclease in a large fraction of the target cells, which raises the chances of unwanted edits occurring. In the two studies, functional off-target genomic changes were not observed, probably owing to the narrow window of activity (a few days) of the Cas9 protein translated from the mRNA cytosolically expressed after viral delivery (both studies used integration-defective recombinant lentiviruses; pictured).

The delivery of mRNA rather than DNA encoding for Cas9 also lowers the risk of integration of the nuclease into the host genome - an obvious safety concern, as prolonged activity of the nuclease would elevate the levels of off-target editing, and uncontrolled integration could disrupt the expression of endogenous genes (still, most viral vectors used for delivery are integration-deficient). But as highlighted by Felix Bubeck and Dirk Grimm in an accompanying News \& Views article, the manufacturing of lentiviral particles packaging RNA coding for the endonuclease and an expression cassette for the RNA guide involves the co-transfection of multiple RNA-encoding plasmids, and persistence of the episomal cassette could lead to it being integrated into the cell's genome.

Another common concern with the viral delivery of foreign proteins such as Cas9 is the ensuing immune responses to both the vector and the protein. When the immunogenicity is substantial, it diminishes the delivery and editing efficiencies, and hence the extent of the therapeutic outcomes (on the first injection if the recipient has pre-existing immunity to the vector or the protein, or on subsequent administration if not). However, the retina and the cornea are immune-privileged tissues (largely because of the blood-retina barrier, the absence of efferent lymphatics and the presence of immunosuppressive factors). Still, in most tissues the transient nature of Cas9 expression and ideally a one-and-done injection should preclude strong immune responses to the therapy.

The fact that modes of local administration to eye tissues are a clinical routine has propelled the development of CRISPR therapies for eye diseases. Local injections (subretinal and intracorneal, in the case of the two studies highlighted here) alleviate the safety and efficacy challenges that come with systemic delivery: the payload preferentially accumulates in first-pass organs (such as the lungs) or in organs that are large or that function as a filter (such as the liver and the spleen). The most researched strategies to favour active delivery over such passive accumulation are the incorporation of tissue-specific or cell-specific molecular tags onto the delivery vehicle or payload, and the development of liposomal and other synthetic vehicles (to avoid viral vectors altogether). But these approaches come with their own challenges.

A one-shot transient approach for therapeutic gene editing is therefore an advantageous proposition on many fronts. And not for your eyes only.

Published online: 12 February 2021 https://doi.org/10.1038/s41551-021-00695-z 